\title{
Vibration Analysis of a New Type of Compliant Mechanism with Flexible-Link, Using Perturbation Theory
}

\author{
N. S. Viliani, ${ }^{1}$ H. Zohoor, ${ }^{2,3}$ and M. H. Kargarnovin ${ }^{3}$ \\ ${ }^{1}$ Department of Mechanical and Aerospace Engineering, Science and Research Branch, \\ Islamic Azad University, Tehran, Iran \\ ${ }^{2}$ Center of Excellence in Design, Robotics, and Automation, Sharif University of Technology, Tehran, Iran \\ ${ }^{3}$ School of Mechanical Engineering, Sharif University of Technology, Azadi Avenue, \\ Tehran 14588-89694, Iran
}

Correspondence should be addressed to N. S. Viliani, navid.viliani@gmail.com

Received 6 December 2011; Revised 8 February 2012; Accepted 24 February 2012

Academic Editor: Slim Choura

Copyright (c) 2012 N. S. Viliani et al. This is an open access article distributed under the Creative Commons Attribution License, which permits unrestricted use, distribution, and reproduction in any medium, provided the original work is properly cited.

Vibration analysis of a new type of compliant parallel mechanism with flexible intermediate links is investigated. The application of the Timoshenko beam theory to the mathematical modeling of the intermediate flexible link is described, and the equations of motion of the flexible links are obtained by using Lagrange's equation of motion. The equations of motion are obtained in the form of a set of ordinary differential equations by using assumed mode method theory. The governing differential equations of motion are solved using perturbation method. The assumed mode shapes and frequencies are to be obtained based on clamped-clamped boundary conditions. Comparing perturbation method with Runge-Kutta-Fehlberg 4, 5th leads to highly accurate solutions, and the results are performed and discussed.

\section{Introduction}

Micro- and nanopositioning is essential technologies that play a significant role in many technical fields such as microbiology, surgery automation in medicine, scanning electron microscopy, and the manipulation of microscale components in microassembly. In recent years, there has been considerable interest in dynamic modeling of the micrometer positioning system based on the compliant mechanism concept.

Unlike conventional mechanisms, compliant mechanism is a monolithic structure that provides the required motion by flexure hinges inherent to the structure and has capacity to be utilized in small-scale applications. 
A foundation work on the flexure hinges was presented by Paros and Weisbord [1]. In modeling and analyzing compliant mechanisms, the pseudo-rigid-body model (PRBM) approach is the almost exclusive tool, that is, currently utilized. The PRBM considers a flexure hinge as a revolute joint with an attached torsional spring. This concept has been introduced and then developed by Midha et al. [5], Howell and Ananthasuresh and Kota [2], Murphy et al. [3], and Brockett and Stokes [4].

A flexure hinge is in fact a complex spring element that can respond to and transmit both rotational and translational motions. Flexure hinges are classified into different profiles such as circular, beam type, corner filleted, parabolic, hyperbolic, elliptical, inverse parabolic and secant flexure hinges [5]. But circular hinges have been widely used because they could be easily manufactured. Paros and Weisbord were the first group to introduce circular flexure hinges. They derived the design equations, including full and simplified equations to calculate compliance of flexure hinges. Rong et al. [6] derived analytical compliance equations of circular flexure hinges which can be reduced to the simplified equation of Paros and Weisbord. Her and Chang [7] used finite element approach to numerically determine the rotational stiffness of circular flexure hinges. Smith et al. [8] studied both the compliance of circular and elliptical flexure hinges. Zhang and Fasse [9] derived empirical equations based on FEA results to predict the compliance of circular flexure hinges.

Lobontiu et al. [10] derived analytical equations to predict the compliances of the corner-filleted flexure hinges along all three axes. Shim et al. [11] derived a kinematic model of a six-DOF parallel micromanipulator for micro-positioning applications. Geo et al. derived a static model for two-DOF compliant mechanism and $x$ deformation of flexure hinges which was considered in these models.

Lobontiu and Garcia [12] analyzed and formulated displacement and stiffness calculations of planar compliant mechanisms with flexure hinges. The formulations were based on strain energy and Castiglione's displacement theorem. Yu et al. [13] presented a kinematic model for three-DOF compliant micromotion stage, and model was derived based on the PRBM concept.

Tian et al. [14] derived the dimensionless empirical equations and graph expressions of three flexure hinges for compliant mechanism.

Clearly, for nanopositioning applications, accuracy and precision of mechanism is the most important. Furthermore, parallel structures have been adopted in many micropositioning devices and are widely used in many applications, and the flexibility of link improves accuracy and some other characteristics of manipulator such as minimizing the energy needed to run the manipulator, increasing speed, reducing the internal stresses and displacement.

Significant achievements, which have driven research on flexible manipulators, were reported by Kanoh et al. [15], Baruh and Tadikonda [16], Book [17], Dwivedy and Eberhard [18], and Tokhi and Azad [19].

Timoshenko beam theory accounts for both the effect of rotary inertia and shear deformation, which are neglected when applied to Euler Bernoulli Beam Theory (EBBT). The predictions of the Timoshenko Beam Theory (TBT) are in excellent agreement with the results obtained from the elasticity equations and experimental results, as they can be seen in the papers by Abramovich and Elishakoff [20], Han et al. [21], and Stephen [22].

In this paper for the first time a new compliant micromotion stage with three flexible intermediate links is presented. The 3RPR links are arranged in parallel, and each link has 2 circular flexure hinges. Each intermediate link has been treated as a Timoshenko beam. Structural dynamic equations of the proposed compliant mechanism are derived based on 


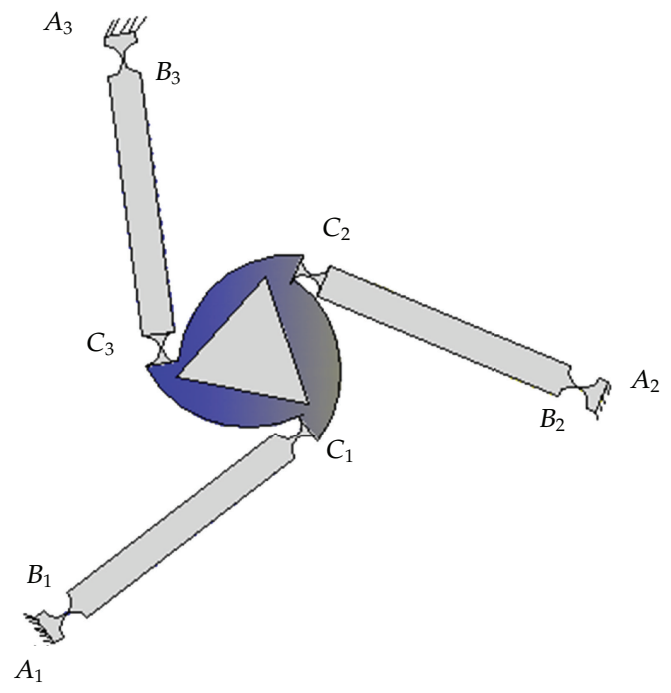

Figure 1: Modeling of parallel micropositioning compliant mechanism.

Lagrange's equations. Also the AMM is adopted to discretize the distributed dynamic system of the manipulator system with flexible links and solved using perturbation method and then results for the deflection of the intermediate link are compared with Runge-Kutta-Fehlberg 4 , 5th. Furthermore, numerical simulations are performed to illustrate and analyze the mode characteristics of the compliant mechanism.

\section{Compliant Micromotion System Configuration}

The micromotion system is proposed as a parallel mechanism that consists of three chains that connect the end effector to a fixed base. The compliant micromotion mechanism is illustrated in Figure 1. The end effector translates along $x$-axis and $y$-axis and rotates about the $z$ axis. The first flexure hinge in each chain is modeled as having 2DOF hinge. Consequently, first hinge assumes that the flexure hinges in the mechanism act like a 1DOF revolute joint and a 1DOF prismatic joint and the second flexure hinge in each chain is modeled as a revolute joint.

In order to facilitate the formulation of kinematics and dynamics, all coordinates are shown in Figure 2. The three chains are 120 degrees apart from each others and mechanism also has a symmetrical configuration and thus is less sensitive to temperature gradient that can change the kinematics of structure due to material expansion or contraction. The $B_{i} C_{i}$ are assumed flexible. The first revolute joint in each chain is active joint, and the other ones are passive joints.

\section{Dynamic Modeling}

In order to obtain the dynamic model of the $i$ th linkage of compliant mechanism, the total kinetic and potential energies are evaluated. The general form of Lagrange's equations is used 


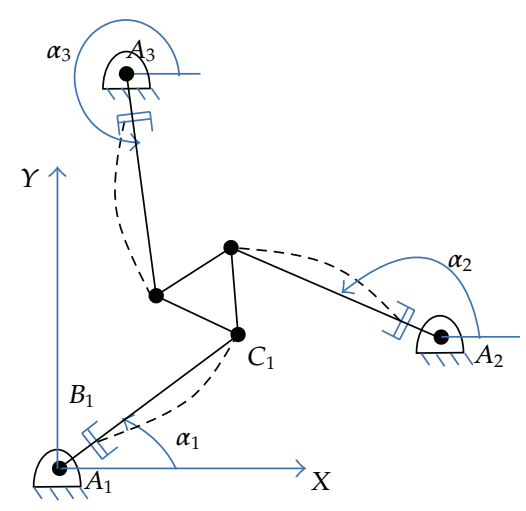

Figure 2: Coordinate system of the 3-RPR parallel compliant mechanism.

to derive dynamic equation of motion for parallel compliant mechanism with three flexible intermediate links.

\subsection{Kinetic and Potential Energy of ith Links}

Each intermediate link can be treated as a beam. In present paper, Timoshenko beam theory was adopted. One of the links is shown in Figure 3. The component $W_{i}(x, t)$ represents the transverse displacement of the $i$ th link at a distance $x$ from the joint coordinate system along the $x$ axis. $\alpha_{i}+\beta_{i}(t)$ represents the angle between the link and the horizontal axis, and $u_{i}(t)$ is the axial deformation of flexure hinges.

The total energy of manipulator system includes the kinetic energy of flexure hinges, flexible intermediate link, and moving platform. The position vector relative to an inertial frame can be written as

$$
\begin{aligned}
r_{i}= & {\left[\left(u_{i}+x+L_{i f l e x}\right) \cos \left(\alpha_{i}+\beta_{i}\right)-W_{i}(x, t) \sin \left(\alpha_{i}+\beta_{i}\right)\right] i } \\
& +\left[\left(u_{i}+x+L_{i f l e x}\right) \sin \left(\alpha_{i}+\beta_{i}\right)+W_{i}(x, t) \cos \left(\alpha_{i}+\beta_{i}\right)\right] j,
\end{aligned}
$$

or matrix form

$$
r_{i}=\left[\begin{array}{c}
\cos \left(\alpha_{i}+\beta_{i}\right)-\sin \left(\alpha_{i}+\beta_{i}\right) \\
\sin \left(\alpha_{i}+\beta_{i}\right) \cos \left(\alpha_{i}+\beta_{i}\right)
\end{array}\right]\left[\begin{array}{c}
\left(u_{i}(t)+x+L_{i f l e x}\right) \\
W_{i}(x, t)
\end{array}\right] .
$$

And derivation of (3.2) is

$$
\dot{r}_{i}=\left[\begin{array}{cc}
\cos \left(\alpha_{i}+\beta_{i}\right) & -\sin \left(\alpha_{i}+\beta_{i}\right) \\
\sin \left(\alpha_{i}+\beta_{i}\right) & \cos \left(\alpha_{i}+\beta_{i}\right)
\end{array}\right]\left[\begin{array}{c}
\left(-W_{i}(x, t) \dot{\beta}_{i}+\dot{u}_{i}(t)\right) \\
\dot{\beta}_{i}\left(u_{i}(t)+x+L_{i f l e x}\right)+\dot{W}_{i}(x, t)
\end{array}\right] .
$$

And velocity of $i$ th intermediate flexible link is:

$$
V_{i \mathrm{link}}^{2}=\left[\dot{u}_{i}(t)-W_{i}(x, t) \dot{\beta}_{i}\right]^{2}+\left[\dot{\beta}_{i}\left(u_{i}(t)+x+L_{i f l e x}\right)+\dot{W}_{i}(x, t)\right]^{2} .
$$




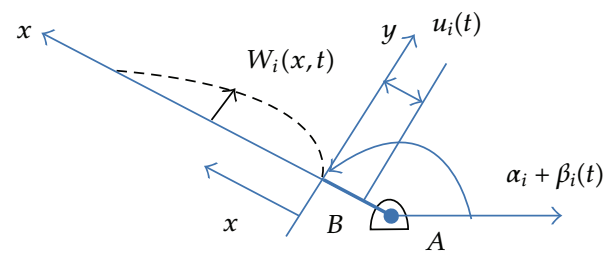

Figure 3: Compliant mechanism with flexure hinge and flexible link.

In which $i=1,2,3$ in the above equations. The kinetic and potential energies have differences by Euler Bernoulli beam theory. The kinetic and potential energies include the effects of bending moment, lateral displacement, rotary inertia, and shear distortion. The total kinetic energy of the intermediate flexible link and its potential energy due to the internal bending moment and the shear force are, respectively, given by

$$
\begin{aligned}
& T=\frac{1}{2} \int_{0}^{L_{i}}\left(\rho A\left(\frac{\partial W(x, t)}{\partial t}\right)^{2}+\rho I\left(\frac{\partial \gamma(x, t)}{\partial t}\right)^{2}\right) d x \\
& V=\frac{1}{2} \int_{0}^{L_{i}}\left(E I\left(\frac{\partial \gamma(x, t)}{\partial x}\right)^{2}+\operatorname{KAG}\left(\left(\frac{\partial W(x, t)}{\partial x}\right)-\gamma(x, t)\right)^{2}\right) d x
\end{aligned}
$$

In which $\gamma(x, t)$ is slope of bending, and $(\partial W(x, t)) / \partial x$ is slope of centerline. The total kinetic energy of system is

$$
T=T_{\text {flex }}+T_{\text {link }}+T_{P}
$$

$T_{P}$ is kinetic energy of moving platform and $T_{\text {link }}$ is kinetic energy of flexible intermediate link and $T_{\text {flex }}$ is kinetic energy of flexure hinges. Then total kinetic energy is written as:

$$
\begin{aligned}
T= & \frac{1}{2} \sum_{i=1}^{3} \int_{0}^{L_{i f l e x}} \rho_{0}\left(\dot{u}_{i}^{2}(t)+\dot{\beta}_{i}^{2}\left(L_{i f l e x}+u_{i}(t)\right)^{2}\right) d x \\
& +\frac{1}{2} \sum_{i=1}^{3} \int_{0}^{L_{i \operatorname{link} k}} \rho_{1}\left[\dot{\beta}_{i}^{2}\left(2 x L_{i f l e x}+u_{i}^{2}(t)+2 u_{i}(t) L_{i}+x^{2}+L_{i f l e x}^{2}+2 L_{i f l e x} u_{i}(t)\right)+\dot{u}_{i}^{2}(t)\right] d x \\
& -\dot{u}_{i}(t) \dot{\beta} Q_{i j}^{2} \delta_{i j}+\frac{1}{2} Q_{i j}^{1} \dot{\delta}_{i j}^{2}+u_{i}(t) Q_{i j}^{2} \dot{\delta}_{i j} \dot{\beta}_{i}+Q_{i j}^{3} \dot{\delta}_{i j} \dot{\beta}_{i}+L_{i f l e x} Q_{i j}^{2} \dot{\delta}_{i j} \dot{\beta}+\frac{1}{2} Q_{i j}^{1} \delta_{i j}^{2} \dot{\beta}^{2} \\
& +\frac{1}{2} m_{p}\left(\dot{x}_{p}^{2}+\dot{y}_{p}^{2}\right)+\frac{1}{2} I_{p} \theta_{p}^{2}+\frac{1}{2} \rho I q_{i j}^{1} \dot{\delta}_{i j}^{2} .
\end{aligned}
$$

In which $\rho_{0}$ and $\rho_{1}$ are mass per unit length of flexure hinges and mass per unit length of $i$ th link, respectively. In addition $I_{p}$ is mass moment of inertia of the platform around the center point $P, m_{p}$ is the mass of the platform, $x_{p}$ and $y_{p}$ are positions of platform along $x$-axis and $y$-axis directions, respectively, and $\theta_{p}$ is the orientation of platform at the mass center $P$. 


\subsection{Potential Energy}

The potential energy of intermediate link expresses the internal energy due to bending and the elastic deformation of the link. According to Timoshenko beam theory, the potential energy of the intermediate link is given as [21]

$$
V_{\text {link }}=\frac{1}{2} \sum_{i=1}^{3} \int_{0}^{L_{\text {ilink }}}\left(E_{i} I_{i}\left(\frac{\partial \gamma(x, t)}{\partial x}\right)^{2}+\operatorname{KAG}\left(\left(\frac{\partial W(x, t)}{\partial x}\right)-\gamma(x, t)\right)^{2}\right) d x
$$

where $E_{i}$ is elastic modulus of the $i$ th link, $I_{i}$ is the second moment of area of the $i$ th link, $G$ is the shear modulus, and $K$ is the shear coefficient. In order to obtain potential energy of flexure hinges, they are modeled as revolute and prismatic joints with constant torsional and translational stiffness $k_{\theta f l e x}$ and $k_{x f l e x}$. All the first flexure hinges in each chain are assumed as a combination of torsional and translational stiffness, and the second flexure hinges are assumed to have just torsional stiffness. Using the formulation presented by Lobontiu, the rotational and translational stiffness of circular flexure hinge can be estimated. Those compliance equations are presented in Appendix A [5, 23].

Potential energy of flexure hinges can be estimated as

$$
V_{\text {flex }}=\frac{1}{2} k_{x f l e x}\left(u_{i}(t)\right)^{2}+\frac{1}{2} k_{\theta_{1 i} \text { flex }}\left(\beta_{i}\right)^{2}+\frac{1}{2} k_{\theta_{2 i} \mathrm{flex}}\left(\theta_{p}\right)^{2} .
$$

In which $k_{\theta_{1} \text { flex }}$ is torsional stiffness of the first flexure hinge, and $k_{\theta_{2} \text { flex }}$ is torsional stiffness of the second flexure hinge. The total potential energy of the system is given as:

$$
\begin{aligned}
& V=\frac{1}{2} \sum_{i=1}^{3} \int_{0}^{L_{i \mathrm{ink} k}}\left(E_{i} I_{i}\left(\frac{\partial \gamma(x, t)}{\partial x}\right)^{2}+\operatorname{KAG}\left(\left(\frac{\partial W(x, t)}{\partial x}\right)-\gamma(x, t)\right)\right) d x \\
&+\frac{1}{2} k_{x \mathrm{flex}}\left(u_{i}(t)\right)^{2}+\frac{1}{2} k_{\theta_{1 i} \mathrm{flex}}\left(\beta_{i}\right)^{2}+\frac{1}{2} k_{\theta_{2 i} \mathrm{flex}}\left(\theta_{p}\right)^{2} .
\end{aligned}
$$

\subsection{Dynamic Equations of Motion}

The governing equations of motion are derived using Lagrange's equations. The Lagrangian is computed using kinetic and potential energy as follows:

$$
L=T-V .
$$

And the Lagrange's equations are given by [24]

$$
\frac{d}{d t}\left(\frac{\partial L}{\partial \dot{q}_{i}}\right)-\frac{\partial L}{\partial q_{i}}=\tau_{i}
$$


In (3.5), the $W_{i}(x, t)$ and $\gamma_{i}(x, t)$ are assumed to share the same time-dependent modal generalized coordinates $\delta_{i j}(t)$ under the following separated forms with the respective mode shape functions $\varphi_{j}(x)$ and $\psi_{j}(x)$ that must satisfy the clamped-clamped boundary conditions

$$
\begin{aligned}
W_{i}(x, t) & =\sum_{j=1}^{r} \delta_{i j}(t) \varphi_{j}(x), \\
\gamma_{i}(x, t) & =\sum_{j=1}^{r} \delta_{i j}(t) \varphi_{j}(x) .
\end{aligned}
$$

Functions $\delta_{i j}(t)$ can be considered as generalized coordinates expressing the deformation of the linkage, and functions $\psi_{j}(x)$ and $\varphi_{j}(x)$ are referred to as assumed modes. The clampedclamped boundary conditions are assumed for the intermediate links. The eigen function of clamped-clamped beam was derived based on Timoshenko beam theory. According to Timoshenko beam theory, position-dependent mode shape function for clamped-clamped beam is selected as [21]

$$
\begin{aligned}
& \varphi_{j}(x)=B_{1} \cosh \left(\frac{p s_{1} x}{l}\right)+B_{2} \sinh \left(\frac{p s_{1} x}{l}\right)+B_{3} \cos \left(\frac{p s_{2} x}{l}\right)+B_{4} \sin \left(\frac{p s_{2} x}{l}\right) \\
& \psi_{j}(x)=C_{1} \cosh \left(\frac{p s_{1} x}{l}\right)+C_{2} \sinh \left(\frac{p s_{1} x}{l}\right)+C_{3} \cos \left(\frac{p s_{2} x}{l}\right)+C_{4} \sin \left(\frac{p s_{2} x}{l}\right) .
\end{aligned}
$$

In which $p$ is the root of the characteristic or frequency equation, and frequency equation of clamped-clamped beam is given as [21]:

$$
\cosh \left(p s_{1}\right) \cos \left(p s_{2}\right)-\frac{p\left(3 b^{2}-r^{2}+p^{2} b^{4}\left(b^{2}+r^{2}\right)\right)}{2\left(1+p^{2} b^{2} r^{2}\right)} \sinh \left(p s_{1}\right) \sin \left(p s_{2}\right)=1,
$$

in which in above equation

$$
r=\frac{R}{L}, \quad R=\sqrt{\frac{I}{A}}, \quad b^{2}=\frac{E I}{\mathrm{KAGL}^{2}} .
$$

Derivation of shape mode can be found in Appendix B. Substituting (3.7), (3.10), and (3.13) into (3.11) and (3.12), we have

$$
\begin{gathered}
Q_{i j}^{1} \ddot{\delta}_{i j}(t)+2 \dot{u}_{i} \dot{\beta}_{i} Q_{i j}^{2}+u_{i} Q_{i j}^{2} \ddot{\beta}_{i}+Q_{i j}^{3} \ddot{\beta}_{i}+l_{i f l e x} Q_{i j}^{2} \ddot{\beta}_{i}-Q_{i j}^{1} \delta_{i j}(t)\left(\dot{\beta}_{i}\right)^{2}+E I \delta_{i j}(t) q_{i j}^{2} \\
+I \rho \ddot{\delta}_{i j}(t) q_{i j}^{1}+\frac{1}{2} \mathrm{KAG} \delta_{i j}(t)\left(-4 M_{i j}+2 Q_{i j}^{5}+2 q_{i j}^{1}\right)=0,
\end{gathered}
$$


in which

$$
\begin{gathered}
Q_{i j}^{1}=\int_{0}^{L_{i l i n k}} \rho_{1}\left(\varphi_{i j}(x)\right)^{2} d x \quad Q_{i j}^{2}=\int_{0}^{L_{i l i n k}} \rho_{1}\left(\varphi_{i j}(x)\right) d x, \quad Q_{i j}^{3}=\int_{0}^{L_{i l i n k}} \rho_{1}\left(\varphi_{i j}(x)\right) x d x, \\
M_{i j}=\int_{0}^{L_{i}}\left(\varphi_{i j}^{\prime}(x) \psi_{i j}(x)\right) d x, \quad q_{i j}^{1}=\int_{0}^{L_{i}}\left(\psi_{i j}(x)\right)^{2} d x, \\
q_{i j}^{2}=\int_{0}^{L_{i}}\left(\psi_{i j}^{\prime}(x)\right)^{2} d x, \quad Q_{i j}^{5}=\int_{0}^{L_{i}}\left(\varphi_{i j}^{\prime}(x)\right)^{2} d x .
\end{gathered}
$$

Equation (3.17) can be rewritten in matrix form as

$$
M \ddot{\delta}+K_{s} \delta+N \ddot{\beta}=F_{\text {cor }}
$$

where $M$ is the modal mass matrix, $K_{s}$ is the modal stiffness matrix, $N \ddot{\beta}$ is the effect of rigidbody motion on elastic vibration of flexible links, and $F_{\text {cor }}$ is Coriolis force.

To obtain equations of motion for EBBT, the value of $K$ (shear coefficient) and rotary inertia in (3.17) should be equal to zero. In this way, the equations of motion for EulerBernoulli Beam Theory can be obtained as follows:

$$
Q_{i j}^{1} \ddot{\delta}_{i j}(t)+2 \dot{u}_{i} \dot{\beta}_{i} Q_{i j}^{2}+u_{i} Q_{i j}^{2} \ddot{\beta}_{i}+Q_{i j}^{3} \ddot{\beta}_{i}+l_{i f l e x} Q_{i j}^{2} \ddot{\beta}_{i}-Q_{i j}^{1} \delta_{i j}(t)\left(\dot{\beta}_{i}\right)^{2}+E I \delta_{i j}(t) q_{i j}^{2}=0 .
$$

For getting frequency equation, we use equation of motion in (3.20) and for considering a constant angular velocity of intermediate link and assuming time-independent axial displacement, one gets the following:

$$
-Q_{i j}^{1}\left(\omega^{2}\right)-Q_{i j}^{1}\left(\dot{\beta}_{i}\right)^{2}+E I q_{i j}^{2}=0
$$

In the above equation $(\ddot{\delta}(\mathrm{t}) / \delta(\mathrm{t}))=-\left(\omega^{2}\right)$, the equivalent frequency may be evaluated by:

$$
\left(\omega^{2}\right)=\frac{-Q_{i j}^{1}\left(\dot{\beta}_{i}\right)^{2}+E I q_{i j}^{2}}{Q_{i j}^{1}} .
$$

Consequently, from (3.22), the natural frequency for the link decreases with angular velocity, such that for some critical values of $\dot{\beta}_{c}=\sqrt{\left(E I q_{i j}^{2} / Q_{i j}^{1}\right)}$ the bending frequency equals zero. For the clamped-clamped boundary conditions, we have

$$
q_{i j}^{2}=\left(\frac{(1+j) \pi}{l}\right)^{4} q_{i j}^{21} .
$$


By substituting (3.23) into (3.22), frequency can be obtained as

$$
\left(\omega^{2}\right)=\frac{E I((1+j) \pi)^{4}}{l^{4} Q_{i j}^{1}} q_{i j}^{21}-\left(\dot{\beta}_{i}\right)^{2}
$$

Therefore, the first term on the right side of (3.24) is the natural frequency expression for nonrotating beam. Equation (3.24) is similar to the results given in [25].

\section{Solution Methodology}

To solve equations of motion, the perturbation method is used, which consists of determining the series convergent to the exact solution. Using Taylor series to expand the terms in (3.17), we have the following:

$$
\frac{d^{2} \delta_{i j}(t)}{d t^{2}}+\frac{\left(a_{1} Q_{i j}^{2}-a_{2} Q_{i j}^{3}+a_{3} Q_{i j}^{2} t-a_{4} Q_{i j}^{2} t^{2}\right)}{Q_{i j}^{1}} \delta_{i j}(t)+\frac{-a_{5} Q_{i j}^{1}+a_{6} Q_{i j}^{4}-a_{7} Q_{i j}^{1} t-a_{8} Q_{i j}^{1} t^{2}}{Q_{i j}^{1}}=0
$$

For mode1, we have

$$
\frac{d^{2} \delta(t)}{d t^{2}}+\frac{\left(\lambda_{1}+\varepsilon\right) \delta(t)-a_{5} Q_{11}^{1}+a_{6} Q_{11}^{4}-a_{7} Q_{11}^{1} t-a_{8} Q_{11}^{1} t^{2}}{Q_{11}^{1}}=0
$$

When $\varepsilon$ is small but is different from zero and $\lambda_{1} \gg \varepsilon$, we suppose that the solution of above equation can be expressed in the following form [26]:

$$
\delta(t, \varepsilon)=\delta_{0}(t)+\varepsilon \delta_{1}(t)+\varepsilon^{2} \delta_{2}(t)+\cdots
$$

Substitute (4.3) into (4.2) and set $\varepsilon=0$, then

$$
\begin{gathered}
Q_{11}^{1} \ddot{\delta}_{0}+\left(\lambda_{1}\right) \delta_{0}(t)-a_{5} Q_{11}^{1}+a_{6} Q_{11}^{4}-a_{7} Q_{11}^{1} t-a_{8} Q_{11}^{1} t^{2}=0 \\
Q_{11}^{1} \ddot{\delta}_{1}+\left(\lambda_{1}\right) \delta_{1}(t)+\delta_{0}(t)=0
\end{gathered}
$$

The general solution of (4.4) can be written as

$$
\delta_{0}(t)=A_{0} \cos \left(t+\beta_{0}\right)+f(t)
$$

where $A_{0}$ and $\beta_{0}$ are arbitrary constants. Therefore (4.5) can be rewritten as

$$
Q_{11}^{1} \ddot{\delta}_{1}+\left(\lambda_{1}\right) \delta_{1}(t)+A_{0} \cos \left(t+\beta_{0}\right)+f(t)=0
$$


Table 1: Compliant mechanism physical parameters.

\begin{tabular}{lcccccc}
\hline$L_{1}=L_{2}=L_{3}(\mathrm{~m})$ & $G(\mathrm{~Pa})$ & $K$ & $A\left(\mathrm{~m}^{2}\right)$ & $I\left(\mathrm{~m}^{4}\right)$ & $\rho\left(\mathrm{kg} / \mathrm{m}^{3}\right)$ & $E\left(\mathrm{~N} / \mathrm{m}^{2}\right)$ \\
\hline 0.05249 & $2.6 e 9$ & 0.83 & $0.18 e-4$ & $1.35 e-11$ & $2.77 e 3$ & $7.1 e 10$ \\
\hline
\end{tabular}

Table 2: Geometrical properties of flexure hinge.

\begin{tabular}{lcccc}
\hline$L_{\text {iflex }}$ & $b$ & $H$ & $t$ & $R_{\text {flex }}$ \\
\hline $6(\mathrm{~mm})$ & $3(\mathrm{~mm})$ & $3(\mathrm{~mm})$ & $0.5(\mathrm{~mm})$ & $3(\mathrm{~mm})$ \\
\hline
\end{tabular}

The homogeneous and particular solutions are

$$
\delta(t)=A_{0} \cos \left(t+\beta_{0}\right)+f(t)+\varepsilon\left(A_{1} \cos \left(t+\beta_{1}\right)+f_{1}(t)\right)+\cdots
$$

And flexible deformation of the first intermediate link for mode 1 can be expressed as

$$
W_{1}(x, t)=\delta(t) \varphi_{11}(x) .
$$

\section{Numerical Results and Discussion}

In the present compliant mechanism, dynamic analysis of parallel compliant mechanism with flexible links is considered. Geometrical and material properties of flexible link are given in Table 1. The geometrical properties for flexure hinges are given in Table 2. Flexures and intermediate links are modeled as aluminum alloy.

The deflection of flexure hinges and intermediate links is determined based on the prescribed motion of the moving platform. To obtain the deflection, velocity, angular velocity, and acceleration, inverse kinematics was used. From inverse kinematics, the displacement and velocity of flexure hinges are given as

$$
\begin{gathered}
u_{i}=\left(x_{p}-\left(l_{1 i f l e x}+L_{i}+l_{2 i f l e x}\right) \cos \left(\alpha_{i}+\beta_{i}(t)\right)+x^{\prime} \cos (\theta)+y^{\prime} \sin (\theta)\right)^{2} \\
+\left(y_{p}-\left(l_{1 i f l e x}+L_{i}+l_{2 i f l e x}\right) \cos \left(\alpha_{i}+\beta_{i}(t)\right)-x^{\prime} \sin (\theta)-y^{\prime} \cos (\theta)\right)^{2}, \\
\dot{u}_{i}=\cos \left(\alpha_{i}+\beta_{i}(t)\right) \dot{x}_{p}+\sin \left(\alpha_{i}+\beta_{i}(t)\right) \dot{y}_{p} .
\end{gathered}
$$

And angular velocities of intermediate links are given as

$$
\dot{\beta}_{i}=\frac{1}{-\left(u_{i}+L_{i}+l_{1 i f l e x}\right)}\left(-\sin \left(\alpha_{i}+\beta_{i}(t)\right) \dot{x}_{p}+\cos \left(\alpha_{i}+\beta_{i}(t)\right) \dot{y}_{p}\right) .
$$




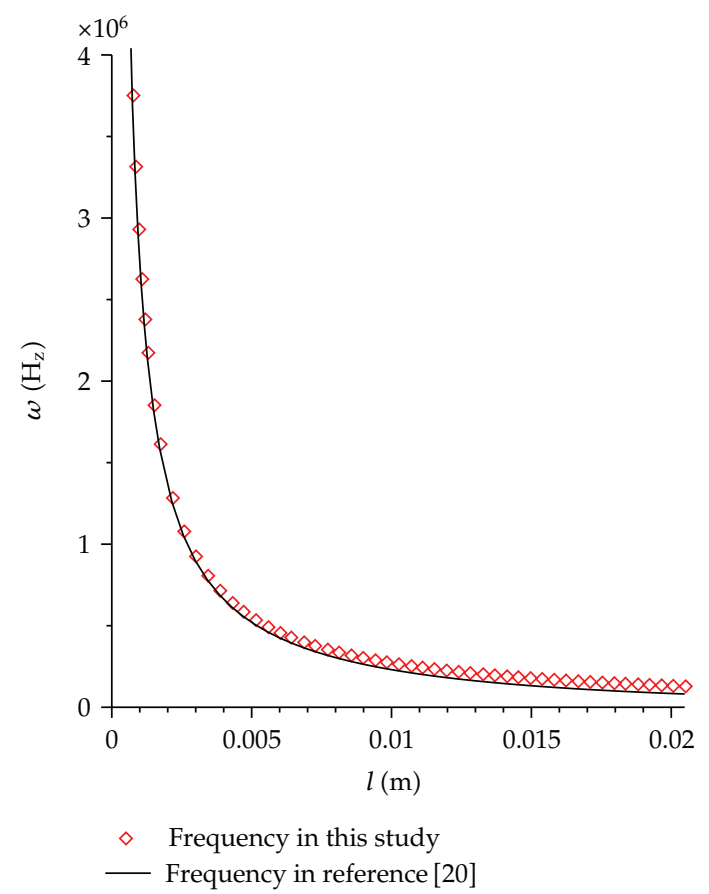

Figure 4: Variation of frequency versus link length.

In this simulation, the moving platform is set to move on a desired trajectory given as

$$
\begin{aligned}
& x_{p}=C_{1}+\frac{x_{f}}{t_{f}} t-\frac{x_{f}}{2 \pi} \sin \left(\frac{2 \pi}{t_{f}} t\right), \\
& y_{p}=C_{2}+\frac{x_{f}}{t_{f}} t-\frac{x_{f}}{2 \pi} \sin \left(\frac{2 \pi}{t_{f}} t\right), \\
& \theta=\text { const },
\end{aligned}
$$

where $x_{f}=0.2 \mathrm{~mm}, t_{f}=1 \mathrm{~ms}, C_{1}=0.039$, and $C_{2}=0.041$. Flexible generalized coordinates in

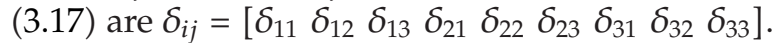

To solve (3.17), initial conditions are adopted $\delta_{i j}=0, \quad \dot{\delta}_{i j}=0$. The deflection of the midpoint of the intermediate links for three modes is obtained by using perturbation method and Runge-Kutta-Fehlberg 4, 5th(RKF4, 5th). To obtain frequency equation for Timoshenko beam, the value of $\dot{\beta}, \ddot{\beta}, \dot{u}, \ddot{u}$ in (3.17) should be equal to zero. By doing so the frequency can be obtained as follows:

$$
\omega^{2}=\frac{E I q_{i j}^{2}+\operatorname{KAG}\left(-2 M_{i j}+Q_{i j}^{5}+q_{i j}^{1}\right)}{Q_{i j}^{1}+I \rho q_{i j}^{1}} .
$$




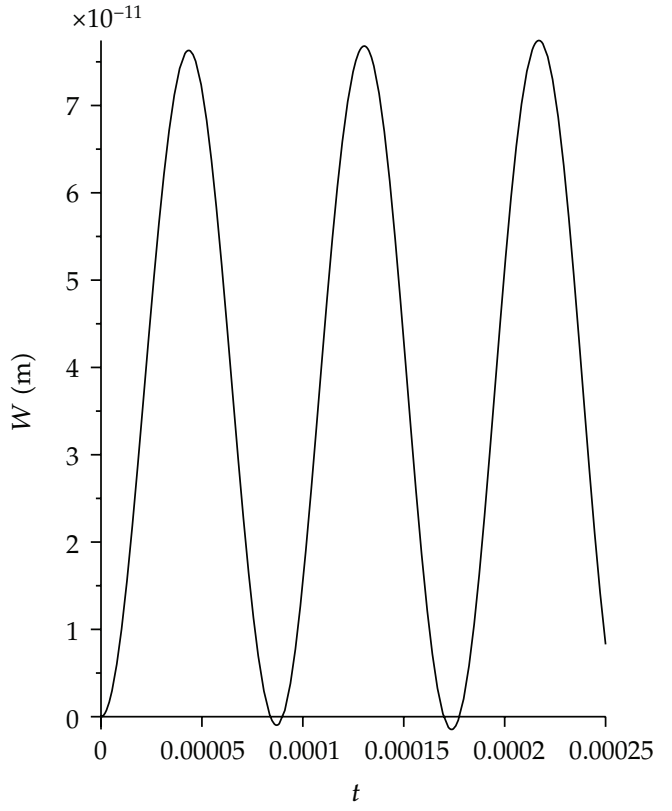

(a)

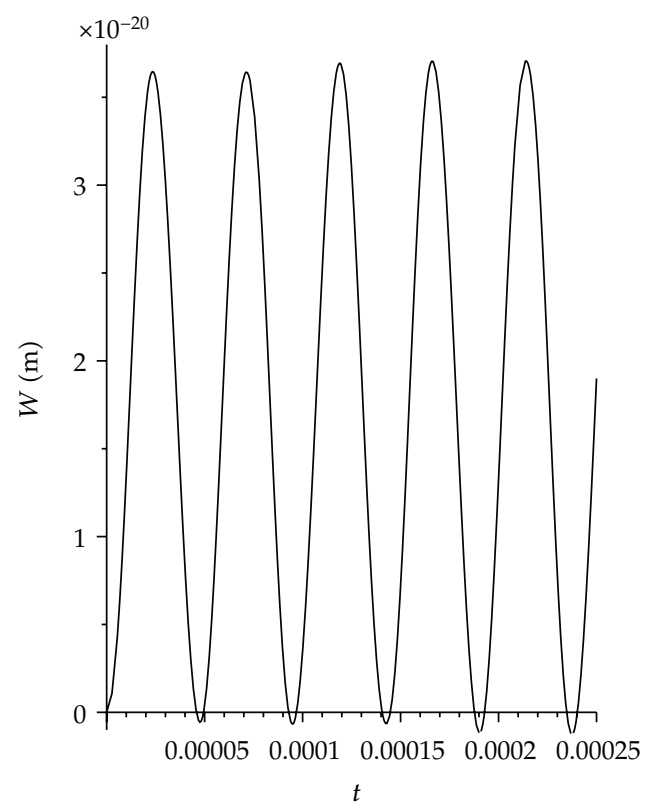

(b)

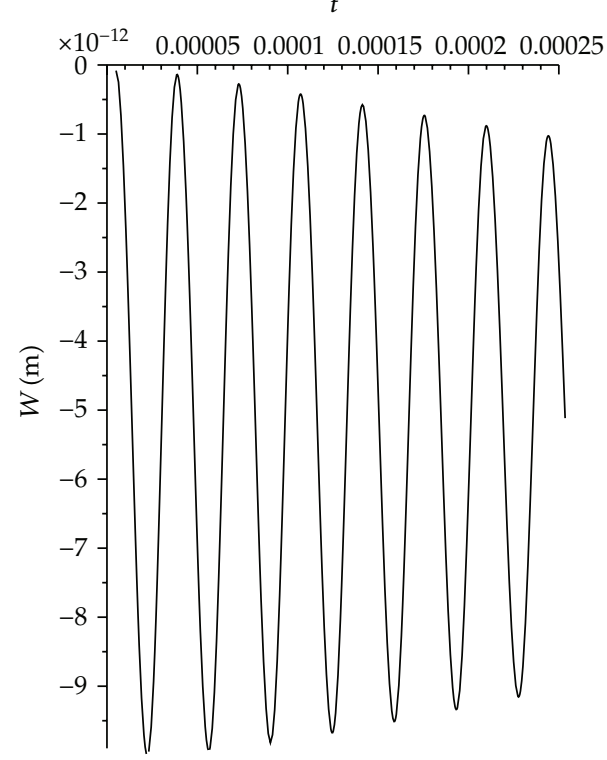

(c)

Figure 5: First three vibration modes of the 1st intermediate link at the midpoint. (a) Mode 1, (b) Mode 2, and (c) Mode 3.

In Figure 4 Variation of frequency versus length of link is illustrated. As it can be seen the trend of variation is similar to the result obtained in the previous study [20].

Figure 5 shows the amplitude of the first three modes of vibration of the first intermediate flexible link at the midpoint and reveals that the amplitude of the first mode vibration is larger than the amplitude of the second mode vibration, and the first mode is sufficiently accurate to describe the vibration of the flexible link. 


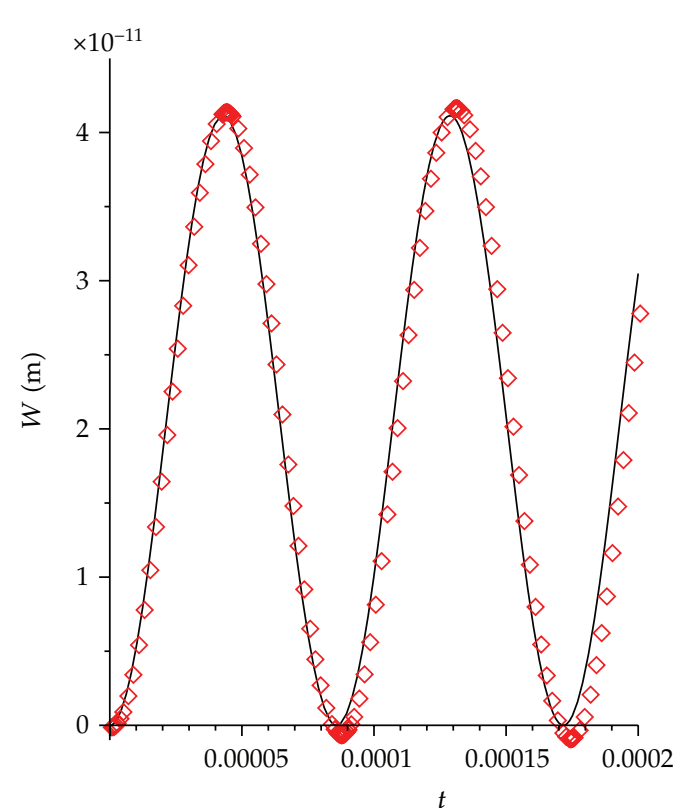

- Perturbation method

$\diamond$ RKF method

(a)

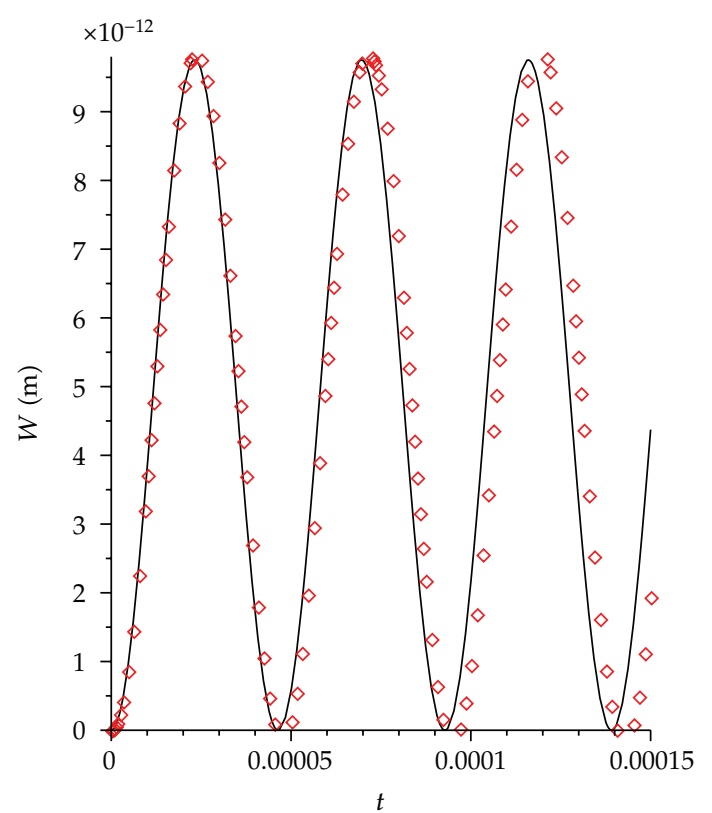

- Perturbation method

$\diamond$ RKF method

(b)

Figure 6: The comparison of results between perturbation method and Runge-Kutta-Fehlberg 4, 5th order. (a) mode 1 and (b) mode 2 .

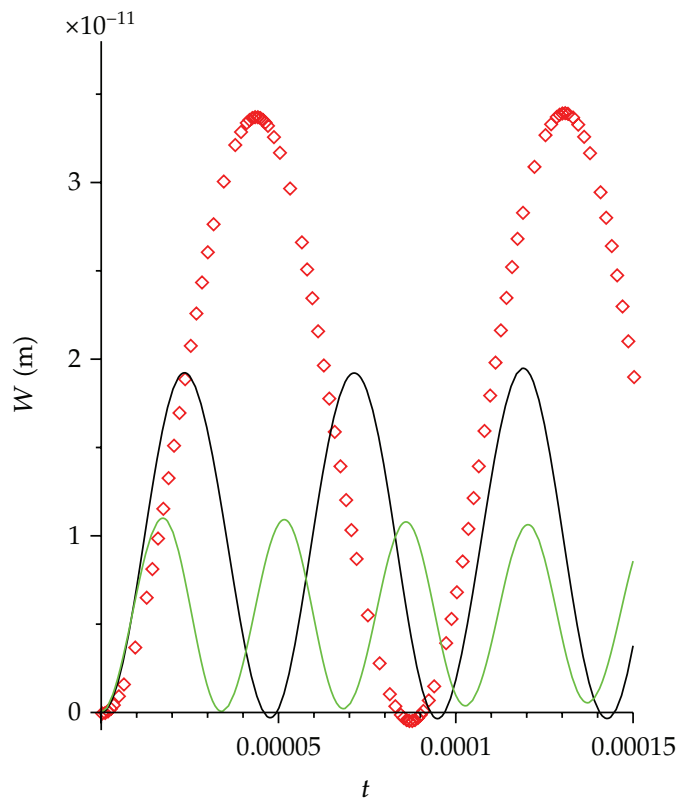

$\diamond \quad$ Mode 1

- Mode 2

Mode 3

Figure 7: First three vibration modes of the 1st intermediate link at the $x=L / 5$. 


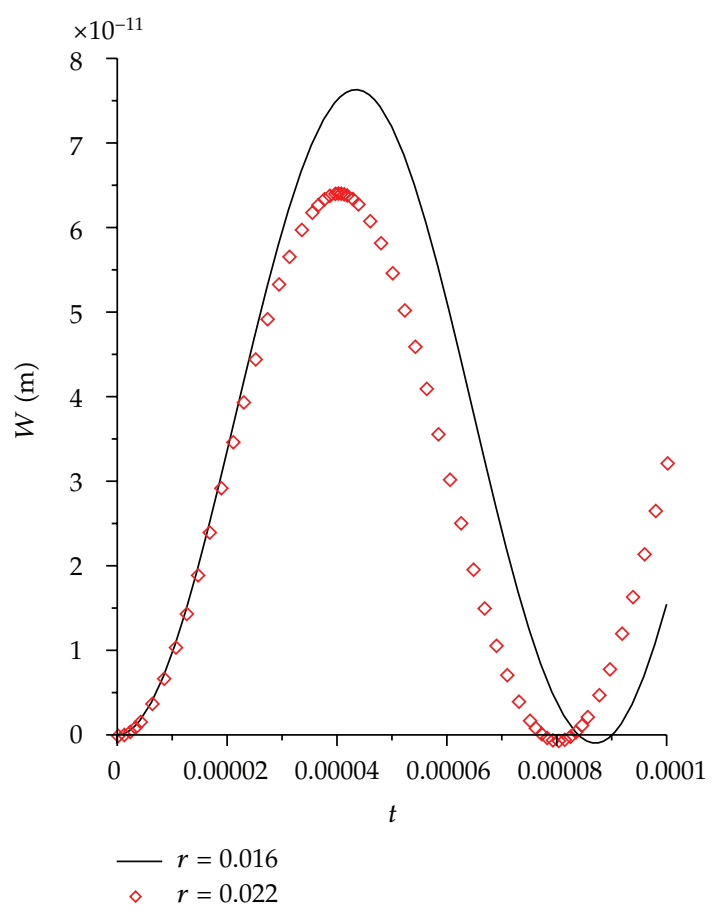

Figure 8: The effect of intermediate link length on deflection.

The comparison of the deflection versus time between results obtained from perturbation method and Runge-Kutta-Fehlberg 4, 5th order has been depicted in Figure 6. It can be observed that there is an excellent agreement between the results obtained from perturbation method with those of Runge-Kutta-fehlberg 4, 5th order method.

Figure 7 shows the amplitude of the first three modes of vibration of the first intermediate link at $x=L / 5$. It reveals that the amplitude of the first mode of vibration is larger than the amplitude of the second and third mode of vibration.

In Figure 8, effect of intermediate link length on deflection is illustrated. From Figure 7 it can be seen that deflection of intermediate link decreases with increasing its height/length ratio.

Figure 9 illustrates deflection of intermediate link at midpoint for Timoshenko beam theory (TBT) and Euler Bernoulli beam theory (EBBT). EBBT is suitable where the link geometry has length to height or aspect ratio greater than 10, approximately. As the height decreases, aspect ratio increases, and EBBT becomes a better model. From Figure 9, it can be seen that by decreasing the thickness (height) of intermediate link, the effects of rotary inertia and shear deformation can be omitted, and behavior of the beam becomes close to EBBT.

In Figure 10 the effect of hinge length on deflection of the midpoint of intermediate link is shown. It shows that when hinge length increases, hinge stiffness decreases (A.2) and the deflection of link increases.

\section{Conclusion}

In this paper, the vibration analysis of a new type of compliant parallel mechanism with flexible links is considered. Perturbation method is adopted for the solution of dynamic equation of motion, and the mode shape functions are selected by modeling intermediate link as Timoshenko beam with clamped-clamped boundary conditions. To derive equations 


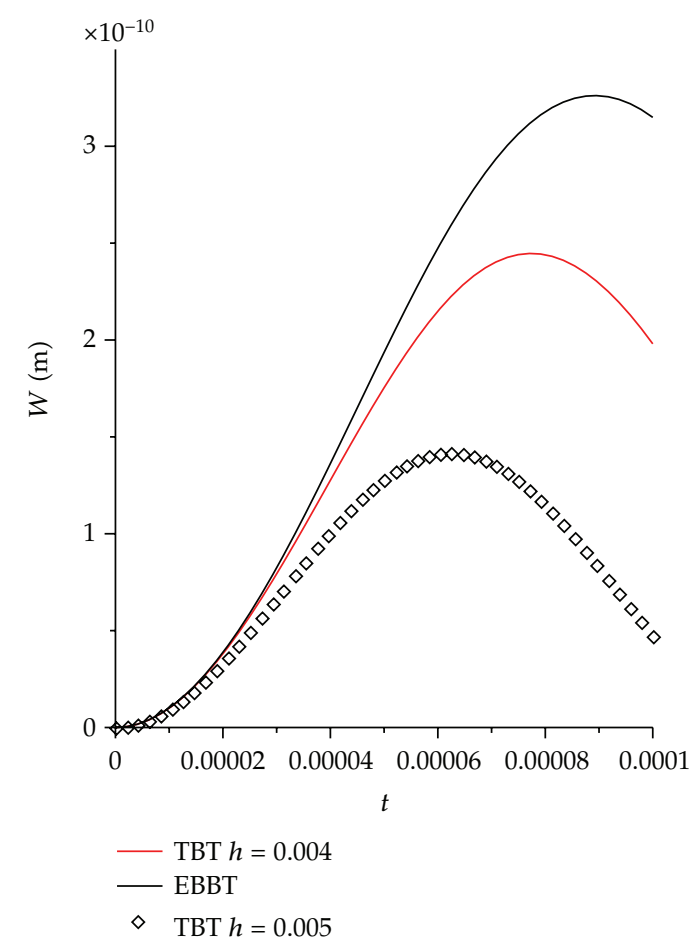

Figure 9: The comparison of results between deflection of intermediate link with TBT and EBBT.

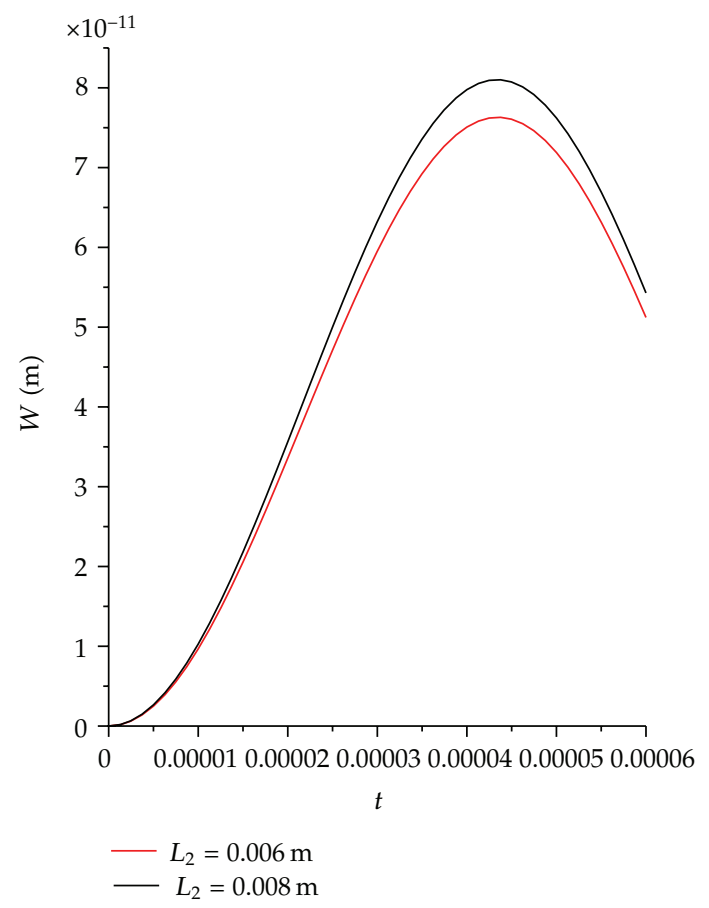

Figure 10: The effect of flexure hinge length on deflection of midpoint of the first intermediate link. 
of motion, the main steps of an energetic deriving procedure based on the Lagrangian method combined with AMM are proposed. The obtained solution using the perturbation method has a very high accuracy compared with Runge-Kutta-Fehlberg 4, 5th order method. Based on numerical results, it is concluded that, for three intermediate links, the elastic deflection is different due to their different base motions, and it has been found that the deflection of intermediate link is decreased, and the frequency is increased with the increase in $r$ (height/length) ratio.

\section{Appendices}

\section{A. Circular Flexure Hinge Compliance Equations}

\section{A.1. Lobontiu [23]}

$$
\begin{aligned}
& \frac{1}{k_{\theta f l e x}}= \frac{\Delta \alpha}{M_{z}}=\frac{24 r}{E b t^{3}(2 r+t)(4 r+t)^{3}} \\
& \times {\left[t(4 r+t)\left(6 r^{2}+4 r t+t^{2}\right)+6 r(2 r+t)^{2} \sqrt{t(4 r+t)} \arctan \left(\sqrt{1+\frac{4 r}{t}}\right)\right], } \\
& \frac{1}{k_{x \mathrm{flex}}}=\frac{\Delta x}{F_{x}}=\frac{1}{E b}\left[\frac{2(2 r+t)}{\sqrt{t(4 r+t)}}\left(\tan ^{-1} \sqrt{1+\frac{4 r}{t}}-\frac{\pi}{2}\right)\right] .
\end{aligned}
$$

\section{A.2. Paros and Weisbord [23]}

$$
\begin{aligned}
& \frac{\Delta \alpha}{M_{z}}=\frac{3}{2 E b r^{2}}\left[\frac{1}{2 \beta+\beta^{2}}\right]\left\{\left[\frac{1+\beta}{\gamma^{2}}+\frac{3+2 \beta+\beta^{2}}{\gamma\left(2 \beta+\beta^{2}\right)}\right]\left[\sqrt{1-(1+\beta-\gamma)^{2}}\right]\right. \\
& \left.+\left[\frac{6(1+\beta)}{\left(2 \beta+\beta^{2}\right)^{3 / 2}}\right]\left[\tan ^{-1}\left(\sqrt{\frac{2+\beta}{\beta}} \times \frac{(\gamma-\beta)}{\sqrt{1-(1+\beta-\gamma)^{2}}}\right)\right]\right\} \\
& \frac{\Delta x}{F_{x}}=\frac{1}{E b}\left[-2 \tan ^{-1} \frac{\gamma-\beta}{\sqrt{1-(1+\beta-\gamma)^{2}}}+\frac{2(1+\beta)}{\sqrt{2 \beta+\beta^{2}}} \tan ^{-1}\left(\sqrt{\frac{2+\beta}{\beta}} \times \frac{\gamma-\beta}{\sqrt{1-(1+\beta-\gamma)^{2}}}\right)\right],
\end{aligned}
$$

in which

$$
\beta=\frac{t}{2} R, \quad \gamma=1+\beta
$$




\section{B.}

To derive frequency and mode shapes of Timoshenko beam, the system of coupled partial differential equations, known as [21]

$$
\begin{gathered}
E I \frac{\partial^{2} \gamma}{\partial x^{2}}+\operatorname{KAG}\left(\frac{\partial W}{\partial x}-\gamma\right)-\rho I \frac{\partial^{2} \gamma}{\partial t^{2}}=0 \\
\rho A \frac{\partial^{2} W}{\partial t^{2}}-\operatorname{KAG}\left(\frac{\partial^{2} W}{\partial x^{2}}-\frac{\partial \gamma}{\partial x}\right)=0 .
\end{gathered}
$$

Above system of differential equations can be decoupled as follows:

$$
\begin{gathered}
E I \frac{\partial^{4} W}{\partial x^{4}}-m R^{2}\left(1+\frac{E}{\mathrm{KG}}\right) \frac{\partial^{4} W}{\partial t^{2} \partial x^{2}}+m \frac{\partial^{2} W}{\partial t^{2}}+\frac{m^{2} R^{2}}{\mathrm{KAG}} \frac{\partial^{4} W}{\partial t^{4}}=0 \\
E I \frac{\partial^{4} \gamma}{\partial x^{4}}-m R^{2}\left(1+\frac{E}{\mathrm{KG}}\right) \frac{\partial^{4} \gamma}{\partial t^{2} \partial x^{2}}+m \frac{\partial^{2} \gamma}{\partial t^{2}}+\frac{m^{2} R^{2}}{\mathrm{KAG}} \frac{\partial^{4} \gamma}{\partial t^{4}}=0 .
\end{gathered}
$$

The last term in (B.2) can be omitted due to its negligible contribution to yield

$$
\begin{gathered}
E I \frac{\partial^{4} W}{\partial x^{4}}-m R^{2}\left(1+\frac{E}{K G}\right) \frac{\partial^{4} W}{\partial t^{2} \partial x^{2}}+m \frac{\partial^{2} W}{\partial t^{2}}=0 \\
E I \frac{\partial^{4} \gamma}{\partial x^{4}}-m R^{2}\left(1+\frac{E}{K G}\right) \frac{\partial^{4} \gamma}{\partial t^{2} \partial x^{2}}+m \frac{\partial^{2} \gamma}{\partial t^{2}}=0
\end{gathered}
$$

Above equations have the following general solutions for transversal and rotational mode shape

$$
\begin{aligned}
& \varphi_{j}(x)=B_{1} \cosh \left(\frac{p s_{1} x}{l}\right)+B_{2} \sinh \left(\frac{p s_{1} x}{l}\right)+B_{3} \cos \left(\frac{p s_{2} x}{l}\right)+B_{4} \sin \left(\frac{p s_{2} x}{l}\right) \\
& \psi_{j}(x)=C_{1} \cosh \left(\frac{p s_{1} x}{l}\right)+C_{2} \sinh \left(\frac{p s_{1} x}{l}\right)+C_{3} \cos \left(\frac{p s_{2} x}{l}\right)+C_{4} \sin \left(\frac{p s_{2} x}{l}\right) .
\end{aligned}
$$

The constants $s_{1}, s_{2}$ can be obtained from

$$
\begin{aligned}
& s_{1}=\sqrt{-\frac{r^{2}+b^{2}}{2}+\frac{1}{2} \sqrt{\left(r^{2}+b^{2}\right)^{2}+\left(\frac{4}{p}\right)}}, \\
& s_{2}=\sqrt{\frac{r^{2}+b^{2}}{2}+\frac{1}{2} \sqrt{\left(r^{2}+b^{2}\right)^{2}+\left(\frac{4}{p}\right)}} .
\end{aligned}
$$


According to the clamped-clamped boundary conditions $(W(0)=W(L)=0, \gamma(0)=\gamma(L)=$ $0)$, we have [21]

$$
\begin{aligned}
& B_{2}=B_{1} \frac{\left(\left(s_{2}^{2}-b^{2}\right) / s_{2}\right)\left(s_{1} /\left(s_{1}^{2}+b^{2}\right)\right) \sin \left(p s_{2}\right)+\sinh \left(p s_{1}\right)}{\cos \left(p s_{2}\right)-\cosh \left(p s_{1}\right)} \\
& B_{3}=-B_{1} \\
& B_{4}=-B_{1} \frac{\left(\left(s_{1}^{2}+b^{2}\right) / s_{1}\right)\left(s_{2} /\left(s_{2}^{2}-b^{2}\right)\right) \sinh \left(p s_{1}\right)+\sin \left(p s_{2}\right)}{\cos \left(p s_{2}\right)-\cosh \left(p s_{1}\right)} .
\end{aligned}
$$

To obtain frequency of TBT, we use assumed mode method and have

$$
\begin{aligned}
W_{i}(x, t) & =\sum_{j=1}^{r} \delta_{i j}(t) \varphi_{j}(x), \\
\gamma_{i}(x, t) & =\sum_{j=1}^{r} \delta_{i j}(t) \psi_{j}(x) .
\end{aligned}
$$

Substitute (B.7) into (B.3), and we have

$$
\begin{gathered}
E I \varphi^{i v}(x) \delta(t)-m R^{2}\left(1+\frac{E}{\mathrm{KG}}\right) \varphi^{\prime \prime}(x) \ddot{\delta}(t)+m \varphi(x) \ddot{\delta}(t)=0, \\
\frac{\ddot{\delta}(t)}{\delta(t)}=-\frac{E I \varphi^{i v}(x)}{m \varphi(x)-m R^{2}\left(1+\frac{E}{\mathrm{KG}}\right) \varphi^{\prime \prime}(x)} .
\end{gathered}
$$

In the above equation $(\ddot{\delta}(\mathrm{t}) / \delta(\mathrm{t}))=-\left(\omega^{2}\right)$, the equivalent frequency may be evaluated by

$$
\omega^{2}=\frac{E I \varphi^{i v}(x)}{m \varphi(x)-m R^{2}\left(1+\frac{E}{\mathrm{KG}}\right) \varphi^{\prime \prime}(x)}
$$

\section{References}

[1] J. M. Paros and L. Weisbord, "How to design flexure hinges," Machine Design, vol. 37, pp. 151-156, 1965.

[2] G. K. Ananthasuresh and Kota, "Designing compliant mechanisms," Mechanical Engineering, vol. 117, no. 11, pp. 93-96, 1995.

[3] M. D. Murphy, A. Midha, and L. L. Howell, "The topological synthesis of compliant mechanisms," Mechanism and Machine Theory, vol. 31, no. 2, pp. 185-199, 1996.

[4] R. W. Brockett and A. Stokes, "On the synthesis of compliant mechanisms," in Proceedings of the IEEE International Conference on Robotics and Automation, pp. 2168-2173, April 1991.

[5] N. Lobontiu, Compliant Mechanisms Design of Flexure Hinges, CRC Press, Fla, USA, 2003. 
[6] Y. Rong, Y. Zhu, Z. Luo, and L. Xiangxi, "Design and analysis of flexure hinge mechanism used in micro-positioning stages," in Proceedings of the International Mechanical Engineering Congress and Exposition, vol. 68, pp. 979-985, ASME, 1994.

[7] I. Her and J. C. Chang, "Linear scheme for the displacement analysis of micropositioning stages with flexure hinges," Journal of Mechanical Design, Transactions of the Asme, vol. 116, no. 3, pp. 770-776, 1994.

[8] S. T. Smith, V. G. Badami, J. S. Dale, and Y. Xu, "Elliptical flexure hinges," Review of Scientific Instruments, vol. 68, no. 3, pp. 1474-1483, 1997.

[9] S. Zhang and E. D. Fasse, "A finite-element-based method to determine the spatial stiffness properties of a notch hinge," Journal of Mechanical Design, vol. 123, no. 1, pp. 141-147, 2001.

[10] N. Lobontiu, J. S. N. Paine, E. Garcia, and M. Goldfarb, "Corner-filleted flexure hinges," Journal of Mechanical Design, vol. 123, no. 3, pp. 346-352, 2001.

[11] J. H. Shim, S. K. Song, D. S. Kwon, and H. S. Cho, "Kinematic feature analysis of a 6-degree-of-freedom in-parallel manipulator for micro-positioning," in Proceedings of the IEEE/RSJ International Conference on Intelligent Robot and Systems, pp. 1617-1623, September 1998.

[12] N. Lobontiu and E. Garcia, "Two-axis flexure hinges with axially-collocated and symmetric notches," Computers and Structures, vol. 81, no. 13, pp. 1329-1341, 2003.

[13] J. Yu, Y. Hu, S. Bi, G. Zong, and W. Zhao, "Kinematics feature analysis of a 3-dof compliant mechanism for micro manipulation," Chinese Journal of Mechanical Engineering, vol. 17, no. 1, pp. 127-131, 2004.

[14] Y. Tian, B. Shirinzadeh, D. Zhang, and Y. Zhong, "Three flexure hinges for compliant mechanism designs based on dimensionless graph analysis," Precision Engineering, vol. 34, no. 1, pp. 92-100, 2010.

[15] H. Kanoh, S. Tzafestas, H. G. Lee, and J. Kalat, "Modeling and control of flexible robot arms," in Proceedings of the 25th conference on Decision and control, pp. 1866-1870, 1986.

[16] H. Baruh and S. S. K. Tadikonda, "Issues in the dynamics and control of flexible robot manipulators," Journal of Guidance, Control, and Dynamics, vol. 12, no. 5, pp. 659-671, 1989.

[17] W. J. Book, "Controlled motion in an elastic world," Journal of Dynamic Systems, Measurement and Control, Transactions of the Asme, vol. 115, no. 2 B, pp. 252-261, 1993.

[18] S. K. Dwivedy and P. Eberhard, "Dynamic analysis of flexible manipulators, a literature review," Mechanism and Machine Theory, vol. 41, no. 7, pp. 749-777, 2006.

[19] M. O. Tokhi and A. K. M. Azad, Flexible Robot ManipulatorssModeling, Simulation and Control, Control Engineering Series 68, The Institution of Engineering and Technology (IET), London, UK, 2008.

[20] H. Abramovich and I. Elishakoff, "Application of the krein's method for determination of natural frequencies of periodically supported beam based on simplified bresse-timoshenko equations," Acta Mechanica, vol. 66, no. 1-4, pp. 39-59, 1987.

[21] S. M. Han, H. Benaroya, and T. Wei, "Dynamics of transversely vibrating beams using four engineering theories," Journal of Sound and Vibration, vol. 225, no. 5, pp. 935-988, 1999.

[22] N. G. Stephen, "The second spectrum of timoshenko beam theory," Journal of Sound and Vibration, vol. 292, no. 1-2, pp. 372-389, 2006.

[23] Y. K. Yong, T. F. Lu, and D. C. Handley, "Review of circular flexure hinge design equations and derivation of empirical formulations," Precision Engineering, vol. 32, no. 2, pp. 63-70, 2008.

[24] H. Baruh, Analytical Dynamics, McGraw-Hill, 1999.

[25] M. A. Trindade and R. Sampaio, "Dynamics of beams undergoing large rotations accounting for arbitrary axial deformation," Journal of Guidance, Control, and Dynamics, vol. 25, no. 4, pp. 634-643, 2002.

[26] A. H. Nayfeh, Introduction to Perturbation Techniques, John Wiley \& Sons, 1981. 


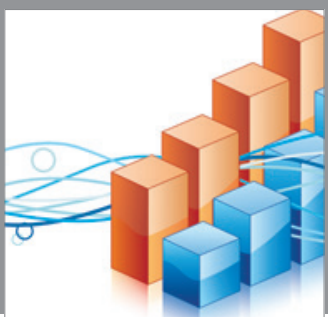

Advances in

Operations Research

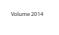

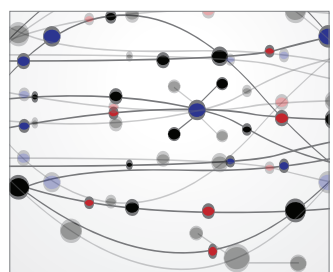

\section{The Scientific} World Journal
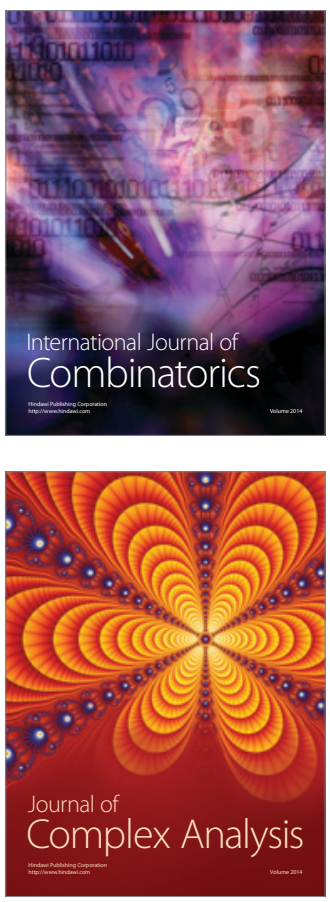

International Journal of

Mathematics and

Mathematical

Sciences
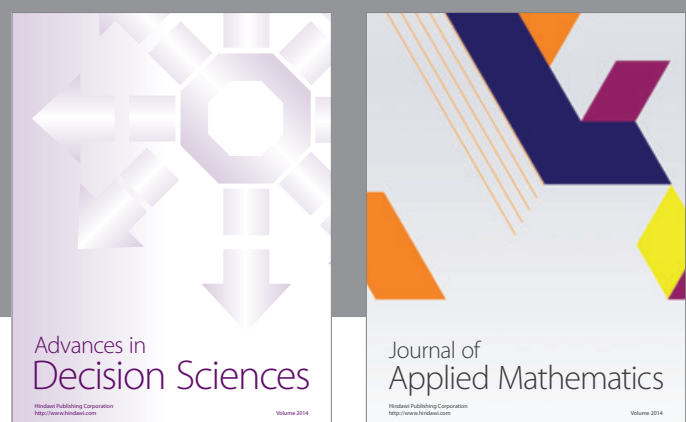

Journal of

Applied Mathematics
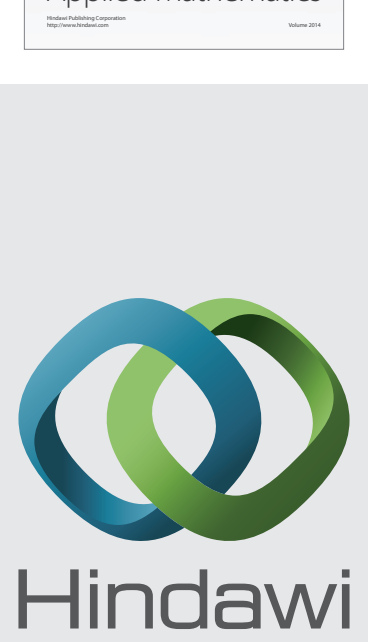

Submit your manuscripts at http://www.hindawi.com
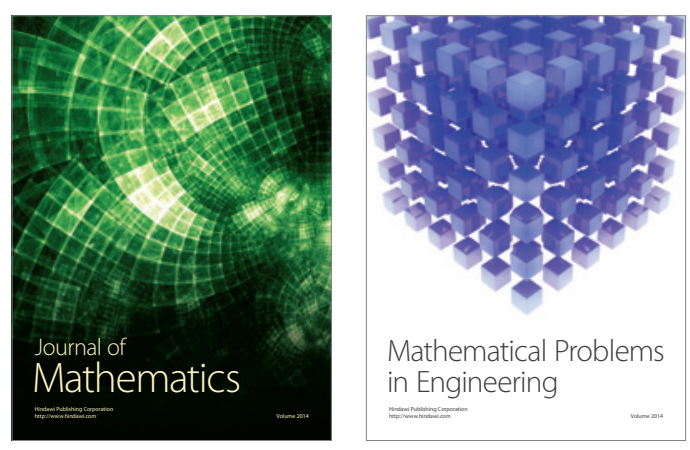

Mathematical Problems in Engineering
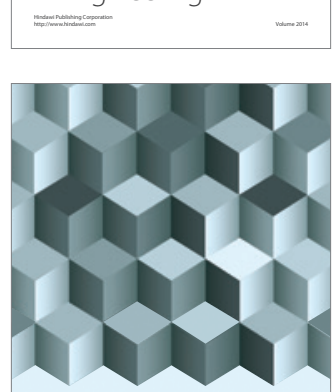

Journal of

Function Spaces
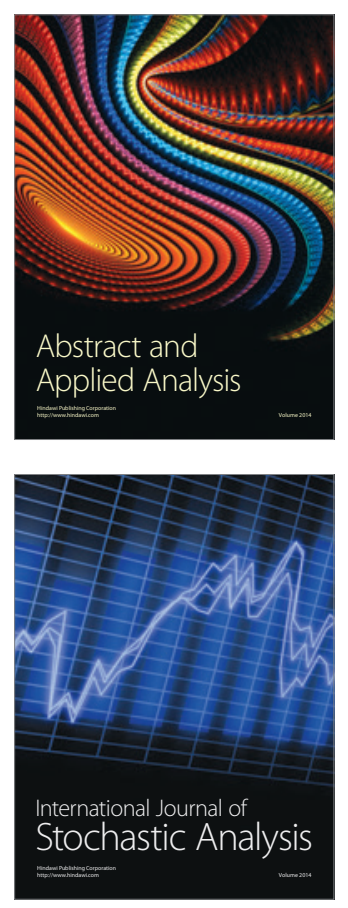

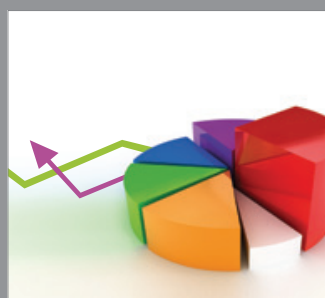

ournal of

Probability and Statistics

Promensencen
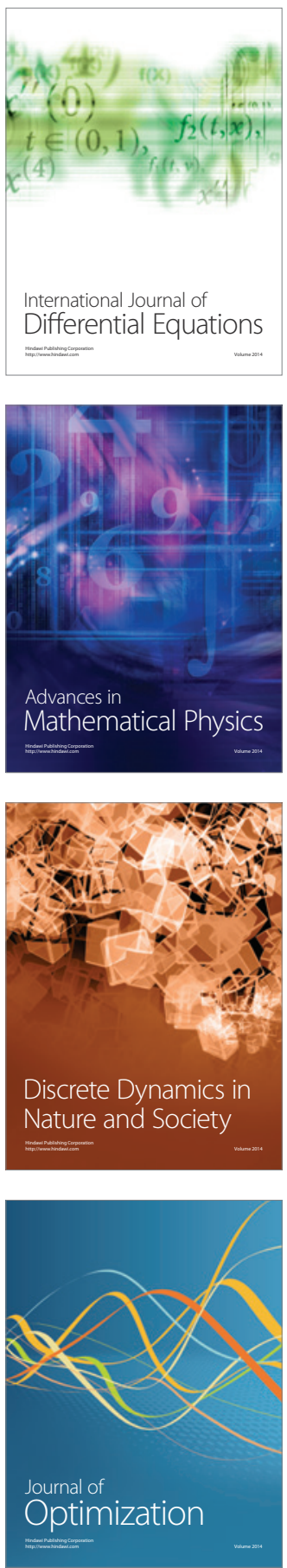